\title{
Technik als Weltbezug, Affordanzen als Reflexionsbegriff
}

\section{Zum Verhältnis von Theorie und Technik in der digitalen Konstellation}

\section{Tim König}

Angenommen: 12. Januar 2022 / Online publiziert: 31. Januar 2022

(C) Der/die Autor(en) 2022

Zusammenfassung Der Artikel beleuchtet das Verhältnis von Technik und Politischer Theorie in der digitalen Konstellation, indem auf Hubigs technikphilosophische Konzeption von Technik als Medium zurückgegriffen wird. Der mediale Charakter technischer Systeme besteht dabei in der Strukturierung des Möglichkeitsraums, in welchem Handlungen erst vorstellbar werden. In diesem Sinne kann Technik als Weltbezug gefasst werden, welcher mit den Akteur:innen eigenen epistemischen Dispositionen in einem dialektischen Verhältnis steht. Anhand des Beispiels digitaler Öffentlichkeit wird illustriert, inwiefern diese Strukturierung epistemischer Möglichkeitsräume durch Technik Gegenstand einer präzisen politiktheoretischen Analyse sein muss. Für die Reflexion des dialektischen Verhältnisses epistemischer Dispositionen und technischer Vermittlung wird weiterhin der Begriff der Affordanzen vorgeschlagen, dessen Mehrwert mit Blick auf die Plattform Twitter aus öffentlichkeitstheoretischer Perspektive illustriert wird. Der Beitrag knüpft somit einerseits an die Diskussion um die Theoretisierung digitaler Technologien an, indem er Perspektiven der Politischen Theorie, der Technikphilosophie und der Science and Technology Studies verbindet. Andererseits greift er Diskussionsstränge um die Epistemologie der digitalen Konstellation auf, indem er auf das Verhältnis zwischen Politischer Theorie und digitalen Phänomenen eingeht. Überlegungen zu der spezifischen Messbarkeit dieser Phänomene knüpfen darüber hinaus an Fragen der Vereinbarkeit von Theorie und Empirie an.

Schlüsselwörter Technikphilosophie · Epistemologie - Digitale Öffentlichkeit · Hubig $\cdot$ Science and Technology Studies

Tim König ( $\triangle)$

Institut für Sozialwissenschaften, Universität Hildesheim, Hildesheim, Deutschland

E-Mail: tim.koenig@uni-hildesheim.de 


\title{
Technology as relation to the world, affordances for reflexivity
}

Considering the relationship between theory and technology in the digital constellation

\begin{abstract}
This article considers the relationship between technology and political theory in the digital constellation by utilizing the conception of technique as a medium. In Christoph Hubig's philosophy, the mediality of technology structures the realm of both possible and conceivable actions. In this sense, technology can be considered as relation to the world, which has a dialectical relationship with an actor's epistemic dispositions. Choosing the digital public sphere as an example, the article shows how this structuration of epistemic realms of possibility through technology is necessarily the subject of any precise theoretical analysis. In order to reflect on this dialectical relationship between epistemic dispositions and technical mediation, the concept of affordances is suggested. By analysing the platform Twitter from a public sphere perspective, the benefits of such a conceptualisation are made apparent. Therefore, the contribution of this article is twofold. For one, it adds to the ongoing discussion on the theorization of digital technologies by bridging the gap between political theory, philosophy of technology and science and technology studies. Second, it reflects on the epistemology of the digital constellation by analysing the relationship between political theory and digital phenomena. Furthermore, it links to questions on the reconciliation of theory and empirical analyses by considering the specific measurability of digital phenomena.
\end{abstract}

Keywords Philosophy of technology · Epistemology - Digital public sphere · Hubig $\cdot$ Science and technology studies

\section{Einleitung}

Die Bewertung technologischen Wandels stellt die Politische Theorie vor besondere Herausforderungen. In jüngeren Jahren manifestiert sich diese Schwierigkeit in der wechselhaften Bewertung des demokratischen Potenzials digitaler Technologie, welche - je nach theoretischer Perspektive und aktuellen politischen wie technischen Entwicklungen - als Wiederbelebung der Demokratie oder ihr Ende, als Ära einer neugefundenen Rationalität demokratischer Regierbarkeit oder totaler, autoritärer Überwachung bewertet wird (Ess 2018; Berg, et al. 2020). Kaufmann und Jeandesboz (2017) identifizieren das Problem der Singularität, welches den digitalen Strukturwandel als eine autonome, politischen und gesellschaftlichen Prozessen äußerliche Kraft verortet, deren Neuartigkeit jede theoretisch herleitbare Kontinuität zu verneinen scheint. Rammert (2016) spricht in diesem Zusammenhang auch von dem technikdeterministischen Paradigma, das Technik als bestimmende Größe gesellschaftlicher Entwicklungen identifiziert. Eine unzureichende Reflexion des komplexen Verhältnisses von Technik und Gesellschaft und ein unterkomplexer Technikbegriff führen so zu einem Mangel an Spezifität in der Analyse des Gegenstands. Besonders deutlich treten diese Probleme in Theorien digitaler Öffentlichkeit hervor, deren Interpretation des digitalen Wandels häufig in eine technikdeterminis- 
tische Richtung tendiert (exemplarisch: Dahlberg 2011; Ritzi 2019; Kaya 2020). Die damit einhergehende Tendenz, Phänomene in bestehende theoretische Gebäude einzuordnen und identische Entwicklungen so gänzlich heterogen zu beurteilen (vgl. Borucki und Oswald 2020), mag aus der Perspektive einer normativen Bewertung gerechtfertigt sein. Es stellt sich jedoch die Frage, inwiefern die Wahl der Theorie selbst bereits die Sicht auf ihren empirisch-analytischen Gegenstand prägt. Alexander (1990) nennt dies das „epistemische Dilemma“: Die Sicht auf ein Phänomen wird unweigerlich durch die spezifische Weltsicht einer angelegten Theorie bestimmt, ohne deren Verallgemeinerungsleistung jede Analyse jedoch auf singuläre, nicht systematisch erfass- und einordbare Beobachtungen beschränkt bliebe. Somit ist es neben dem Verhältnis von Gesellschaft und Technik auch das Verhältnis von Technik und Theorie, welches der Reflexion bedarf, um sicherzugehen, dass die angelegte Theorie nicht notwendigerweise zu einem Mangel an Spezifizität in der Analyse führt.

Einen Ansatz, um diese Reflexionsebene einzuholen, bietet der Affordanzbegriff. ${ }^{1}$ Als soziomaterialistisch-relationale Perspektive auf Technik hat das Konzept der Affordanzen das Potenzial, Dualismen zwischen Subjekt und Objekt, Struktur und Agency, technologischem Determinismus und Voluntarismus zu überwinden und Blackboxing-Effekte in der Analyse von Technik und Gesellschaft aufzulösen (Faraj und Azad 2013). Auch neuere Arbeiten zur „digitalen Konstellation“ greifen auf den Begriff der Affordanzen zurück, um die spezifische Situiertheit digitaler Phänomene einerseits in ihrer Materialität, andererseits in ihrer sozialen und politischen Geformtheit zu analysieren (Hofmann 2019b; Berg et al. 2020). So kann es gelingen, den Formwandel der Demokratie nicht als einseitig-kausalen Prozess anzusehen, bei dem digitale Technik wahlweise durch gesellschaftliche Veränderungen geprägt wird oder diese bedingt, sondern als ,umfassende[n], vielgestaltige[n] Prozess, in dem sich Gesellschaft und Technik in einer konkreten Weise dynamisch und fortlaufend in Beziehung setzen" (Berg et al. 2020, S. 182). Hofmann (2019a, b) verwendet darüber hinaus die Luhmann'sche Unterscheidung von Medium und Form, um die Kontingenz technischer Entwicklungen hervorzuheben und diese historisch zu verorten. Jedoch verpassen es diese Ansätze zu reflektieren, inwiefern die neuen „Möglichkeitsräume“ digitaler Technik (Hofmann 2019b, S. 36; Berg et al. 2020, S. 183) auch nach einer Reflexion ihrer theoretischen Verortung verlangen. So ist unklar, wie theoretische Zugänge auch angesichts sich wandelnder epistemischer Möglichkeitsräume fähig bleiben können, ihrem Gegenstand angemessene Analyseergebnisse zu liefern. Um weder in einen unspezifischen Theorieperspektivismus noch in Technikdeterminismus zu verfallen, scheint es daher notwendig, mittels einer geschärften Analytik das zu entwickeln, was als eine den Funktionslogiken digitaler Phänomene angemessene Perspektive gefordert wird (vgl. Borucki et al. 2020). Erst so kann zwischen den Ansprüchen von analytischer Spezifität und theoretischer Anschlussfähigkeit vermittelt werden.

Der vorliegende Artikel trägt auf zweierlei Weise zur aktuellen Diskussion bei. Zum einen wird an Schnittstellen der Politischen Theorie, der Science and Tech-

\footnotetext{
1 Dieser wird auch von Kaufmann und Jeandesboz (2017) für die Analyse digitaler, stets in Wechselwirkung mit politischen und gesellschaftlichen Konfigurationen stehender Phänomene vorgeschlagen.
} 
nology Studies und der Technikphilosophie angeknüpft, um bestehende Konzepte und Überlegungen zu Theoriebildung in der digitalen Konstellation zu erweitern. Dies betrifft insbesondere eine technikphilosophische Erschließung des Begriffs des Möglichkeitsraums von Technik und die systematische Verbindung des Begriffs der Affordanzen mit der Medium-Form-Unterscheidung. So soll zum anderen ein Reflexionsraum eröffnet werden, welcher die spezifischen epistemischen Bedingungen politiktheoretischer Analysen in der digitalen Konstellation anhand von Theorien digitaler Öffentlichkeit in den Fokus nimmt. Der vorliegende Artikel soll so Lücken in den bestehenden Arbeiten zur Epistemologie der digitalen Konstellation schließen und begriffliche Vorarbeiten für zukünftige empirisch-analytische Untersuchungen leisten. Dazu werde ich auf Christoph Hubigs Konzeption von Technik als Medium zurückgreifen, mittels derer Technik als Handlungen strukturierender Möglichkeitsraum und somit als Weltbezug verstanden werden kann. Den Mehrwert dieser Konzeptualisierung technischer Möglichkeitsräume demonstriert Seibel (2016) in seiner Analyse kybernetischen Regierungshandelns, welches sich an eben jenen Vorstellungen der Verfügbarkeit von Regierungstechnologien mitherausbildete. Auch in Hofmanns (2019b) Überlegungen zu den performativen Rahmenbedingungen technischer Formationen wird Hubigs Begriffsschema strukturierter Möglichkeitsräume verwendet, jedoch nicht systematisch für die Theoriebildung erschlossen. Um diese Lücke zu schließen, werde ich im Anschluss an die Vorstellung des Technikbegriffs zeigen, wie dieser Möglichkeitsraum politiktheoretische Analysen digitaler Öffentlichkeiten mitstrukturiert und so die Reflexion des Verhältnisses von Theorie und Technik notwendig macht. Dies betrifft insbesondere die Lesart des Technikdeterminismus, die spezifische Messbarkeit digitaler Phänomene und die Möglichkeit des Theoriedeterminismus. Für eine Neubetrachtung wird das Konzept der Affordanzen als Reflexionsbegriff eingeführt, um sowohl die Materialität technischer Systeme als auch die Handlungsfähigkeit ihrer Nutzer:innen zu berücksichtigen. In der Vereinbarung der Medium-Form-Unterscheidung mit dem Begriff der Affordanzen wird sich zeigen, wie ein solcher Ansatz die eigene Theorieperspektive an der Spezifität seines Untersuchungsobjekts reflektieren kann. Schlussendlich wird die so entwickelte reflexive Epistemologie am Beispiel politischer Öffentlichkeitstheorie und der Plattform Twitter skizziert.

\section{Die Medialität von Technik}

Alle unsere Welt- und Selbstbezüge sind vermittelt, sowohl in ontologischer als auch in epistemischer Hinsicht (Hubig 2006, S. 145)

Soll Theoriebildung in der digitalen Konstellation sowohl einer technikdeterministischen wie auch einer theorieperspektivistischen Lesart entgehen, braucht es zuvorderst einen Technikbegriff, welcher die spezifische Vermittlungsleistung digitaler Technologie reflektiert. Ein Begriff von Technik als zweckgebundenem Mitteleinsatz, wie etwa Webers „Inbegriff der Mittel“ (Weber 2002, S. 32), welcher der Rationalisierung ihr vorgelagerter Prozesse dient, kann dies nicht abbilden, bleibt er doch auf die einseitige Formung technischer Systeme durch die Vorstellungen menschli- 
cher Akteur:innen beschränkt. So vermag es ein solches Technikverständnis nicht zu reflektieren, inwiefern Technik selbst den Möglichkeitsraum strukturiert, in dem ihr Einsatz vorstellbar wird - und so definiert, welche Handlungen überhaupt als möglich gelten. ${ }^{2}$ Aber auch ein deterministisches Verständnis von Technik, welches von einer einseitigen Formung der Handlungsfähigkeit menschlicher Akteur:innen durch Technik ausgeht, verkennt, dass diese immer vor dem Hintergrund spezifischer Vorstellungen von Mittel, Zweck und Machbarkeit intentional geschaffen wurden (vgl. Rammert 2016, S. 22). In Abgrenzung zu solchen Verständnissen von Technik greife ich stattdessen auf Christoph Hubigs technikphilosophische Konzeption zurück, die Technik ,als System der Dienlichkeit und Herbeiführbarkeit, als Ermöglichung des Gelingens instrumenteller Vollzüge“, mithin also als „Potenzialfunktion“ für konkrete Handlungszusammenhänge versteht (Hubig 2013, S. 121). In seiner dialektischen Technikphilosophie untersucht Hubig eben diese Möglichkeitsräume, indem die Performativität und Disponibilität von Technik reflexiv erschlossen werden (vgl. Hubig 2006, S. 12f.).

Zur Theoretisierung der Potenzialität von Technik verwendet Hubig in Anlehnung an Luhmann den Begriff des Mediums. ${ }^{3}$ Luhmann versteht unter einem Medium den Bestand an lose gekoppelten Elementen, welcher die Herausbildung konkreter Formen ermöglicht, ohne sich dabei selbst zu verbrauchen. Das Medium bildet das Substrat, durch deren feste Kopplung identifizierbare Formen entstehen. Es besteht dabei nicht aus physischen, systemexternen Eigenschaften, sondern wird durch das System, das es verwendet, selbst erzeugt. Für Sprache, die als Grundlage für weitere Medienbildungen wie Wahrheit, Macht oder Geld dient, stellt sich diese Medialität als feste Kopplung zu einzelnen Wörtern und Sätzen dar. Diese setzt zwar die physische Möglichkeit der Laut- oder Schrifterzeugung voraus. Es ist jedoch das Kommunikationssystem, welches Sprache als Medium produziert und reproduziert, damit mögliche Formen vorgibt und so den Sinnaustausch zwischen Kommunikationsteilnehmenden mittels dieser Formen ermöglicht (Luhmann 2015, S. 53 f.).

Während Luhmann Technik jedoch nicht als Medium, sondern als „Entlastungsvorgang" sieht, welcher mittels präziser Konditionierung komplexere Systembildungen ermöglicht (Luhmann 2015, S. 197) und sich so dem Weberschen Technikbegriff annähert, sieht Hubig technische Systeme selbst als Medien, deren lose Kopplungen feste Formen annehmen können:

Im Gegensatz zu Luhmann ist Technik freilich nicht blo $\beta$ als feste Kopplung zum Zweck des ,Kontingenzmanagements“ der Systeme zu erachten, denn eine solche feste Kopplung betrifft nur den Charakter der Mittel als hinreichenden Bedingungen der Realisierung von Zwecken. Vielmehr müssen technische Systeme überhaupt auch als Medien, also als (ihrerseits geformte) lose Kopplungen verstanden werden, die den zielführenden Einsatz von Mitteln ermöglichen. (Hubig 2013, S. 122)

\footnotetext{
${ }^{2}$ Zur vertieften Diskussion um eine solche „technizistische Verkürzung des Handelns“ vgl. Hubig 2006, Kap. 4.

${ }^{3}$ Hubig bezieht sich zwar auf Luhmanns Begriff des Mediums als Menge loser Kopplungen, kritisiert diesen jedoch für seinen „Medienmaterialismus“, welcher es versäumt, den dialektischen Charakter innerer und äußerer Medialität als „,erfahrenen Widerspruch“ zu reflektieren (Hubig 2006, S. 160).
} 
Über die Konzeptualisierung von technischen Systemen als Medien liegt der Fokus der Analyse bei Hubig dabei nicht wie bei Luhmann auf sozialen Systemen, sondern auf der Art und Weise, wie Technik deren Möglichkeiten der Welterschließung strukturiert. Technische Systeme sind dabei die ,realen[n] Strukturen und Ordnungen“, die Handlungen ermöglichen und durch ihre Medialität Möglichkeitsräume grundlegend strukturieren (Hubig 2006, S. 156). Da Technik sich für Hubig nicht in der „Realtechnik“ der Maschinen erschöpft, sondern sich ebenso auf die Intellektualtechnik mit ihren Codes (etwa von wahr/unwahr) und die Sozialtechnik als Ermöglichung von Tauschhandlungen (etwa im Geldverkehr) erstreckt, wird sie zur grundlegenden Kategorie, welche die „Rahmenordnung“ sozialer Interaktion vorgibt (ebd.). Es ist dieser mediale Charakter von Technik, welcher sich in technischen Systemen konkretisiert und die ,[...] bestimmte Art und Weise aus[macht], in der die realen, intellektualen und sozialen Möglichkeitsräume strukturiert sind.“ (ders., S. 155).

Diese Medialität von Technik besteht dabei aus drei Ebenen: dem Möglichkeitsraum, dem Wirklichkeitsraum und der instrumentellen Handlung. Möglichkeits- und Wirklichkeitsraum weisen jeweils eine ,innere“, vorgestellte Medialität und eine „äußere“, im Realitätsvollzug erfahrene Medialität auf (Hubig 2013, S. 121). Erst diese beiden Ebenen mit ihrer je inneren und äußeren Medialität ermöglichen die instrumentelle Handlung, die spezifische Zwecke realisiert. So beschreibt die erste Ebene die allgemeine Konzipierung, ,,den Möglichkeitsraum der Realisierung möglicher Zwecke“, dessen innere Medialität aus den „epistemischen Möglichkeiten, disponible Ursachen zu unterscheiden“ besteht (ebd.). Die äußere Medialität dieses Möglichkeitsraums besteht in der ,notwendig vorauszusetzende[n] (technische[n]) Möglichkeit einer Trennung jener Dispositionen“, also der realen Möglichkeit der Identifikation (vorgestellter) „Ursachen“ (ebd.). Die zweite Ebene der Medialität von Technik besteht nach Hubig in den „Wirklichkeitsräume[n] der Realisierung möglicher Zwecke“ (ebd.), welche unter der Maßgabe des Möglichkeitsraums technische Systeme schaffen. Ihre innere Medialität besteht in dem Katalog konkreter Funktionsideen und Erwartungen der Konstrukteur:innen, Entwickler:innen und Nutzer:innen, welche sich wiederum als äußere Medialität in den „Infrastrukturen der technischen Systeme“ niederschlagen (ders., S. 122). Während die erste Ebene also Technik als Potenzialfunktion beschreibt, beschreibt die zweite Ebene deren zweckgerichtete Umsetzung als Realfunktion - ihre „Performanz“ (ebd.). Auf der dritten Ebene schließlich werden in Form instrumentellen Handelns die möglichen Mittel-Zweck-Relationen des Wirklichkeitsraums aktualisiert und „die Erfahrung der Differenz zwischen vorgestelltem und realisiertem Zweck gezeitigt, über die die Technik als Medium eine ,Spur von ... in Gestalt von unerwarteten (positiven oder negativen) Effekten hinterlässt.“ (ebd.) In der instrumentellen Handlung korrigiert sich somit das Verhältnis der inneren und äußeren Medialität nicht nur auf der Ebene der Performanz, also im Verhältnis von Funktionsideen und Erwartungen zu der real existierenden Infrastruktur, sondern auch auf Ebene des Möglichkeitsraumes, in dem epistemische Dispositionen angesichts ihrer technisch vermittelten Unterscheidbarkeit beständig korrigiert werden und der so erst festlegt, welche Zwecke überhaupt als technisch realisierbar, als möglich, gelten. Aus diesem dynamischen Verhältnis von innerer und äußerer Medialität auf Ebene des Möglichkeits- und Wirklichkeits- 
raumes bedingt sich somit der dynamische Charakter von Technik, welcher - im Gegensatz zum Verständnis von Technik als „Inbegriff der Mittel“ - nie einer abschließenden Vorstellung unterliegen kann (ders., S. 121). Die innere Medialität als Voraussetzung unserer Handlungsplanung ,wächst umgekehrt proportional zu den Einschränkungserlebnissen, über die wir etwas über ihre Möglichkeit erfahren.“ (Hubig 2006, S. 158).

Technik stellt eben eine Menge an lose gekoppelten Elementen, ein Medium, dar, deren Aktualisierung in routinisierten Handlungen diese Elemente fest koppeln, also die Form innerhalb des Mediums herstellen, ohne damit die potenziellen Möglichkeiten weiterer Kopplungen (und damit Handlungen) zu begrenzen. ${ }^{4}$ Die Mittel und Zwecke dieser Handlungen sind dabei stets korrelativ: Zwecke lassen sich nur auf Grundlage dessen bestimmen, was nach Maßgabe der inneren Medialität von Möglichkeits- und Wirklichkeitsraum als „,machbar“ erscheint. Mittel zur Realisierung dieser Zwecke erscheinen nur insofern als solche, wie sie auf Grundlage der äußeren Medialität als deren technisch vermittelte Ermöglichung erscheinen. Anders gesagt: Es erscheint machbar, was möglich erscheint und es erscheint möglich, was machbar erscheint. Die Erfahrung der Differenz zwischen dem, was als Zweck innerlich konzipiert und äußerlich mithilfe technischer Mittel realisiert werden kann erlaubt dabei die Optimierung und Verstetigung technischer Systeme (Hubig 2013, S. 122). Es ist also die stets technisch vermittelte Realisierbarkeit von Zwecken mit ihrer je spezifischen Verbindung von Ermöglichung und Einschränkung, welche reflexiv auf die Konzeption der Zwecke selbst zurückwirkt. Und dies gilt nicht nur auf der Ebene der Performanz von Technik, also der Ermöglichung instrumenteller Handlungen, sondern auch epistemisch, da Technik strukturiert, welche Handlungen überhaupt vorstellbar werden (vgl. auch Seibel 2016, S. 35). Die Medialität technischer Systeme besteht auf einer epistemischen Ebene also darin, dass sie kontingente Handlungen in einem technisch vermittelten (äußeren) Möglichkeitsraum anbietet, welcher wiederum auf einem hypothetisch konzeptualisierten (inneren) Möglichkeitsraum basiert. Real existierende technische Systeme sind durch diesen Möglichkeitsraum informiert, wirken durch spezifische Einschränkungs- und Ermöglichungserfahrungen in der instrumentellen Handlung jedoch wiederum auf diesen zurück. In diesem Sinne wird Technik zu einem Weltbezug, deren Mittel nicht nur Steuerung, sondern auch die Aktualisierung der epistemischen Dispositionen, welche die Vorstellung möglicher Handlungen strukturieren, erst ermöglichen (Hubig 2011, S. 5). In dem von Hubig verwendeten weiten Technikverständnis (vgl. Hubig 2006, S. 27 f.) erscheint Technik so als Vermittlungsleistung, welche unsere Wahrnehmung grundlegend strukturiert: „Jenseits unserer theoretischen und praktischen Weltbezüge, die wir technisch realisieren, können wir uns keine Welt vorstellen.“ (Hubig 2006, S. 15). Erst das dialektische Verständnis der Beziehung von innerer

\footnotetext{
${ }^{4}$ Hubig konzeptualisiert die Unterscheidung von Medium und Form dabei, im Gegensatz zu Luhmann, „eher [als] ein Dispositionsgefälle“ (Hubig 2006, S. 156). Er konstatiert jedoch, ebenso wie Luhmann, dass Medialität nie gänzlich, sondern immer nur anhand konkreter Aktualisierungen in Formen fassbar ist. Realität kann somit als „Gesamtheit der Formen, mit denen wir interagieren“ verstanden werden (Hubig 2006, S. 159f.).
} 
und äußerer Medialität der Möglichkeits- und Wirklichkeitsräume erlaubt es uns, den technischen Aspekt dieser Weltbezüge freizulegen.

\section{Theorien digitaler Öffentlichkeit und die Möglichkeitsräume digitaler Technik}

Mit fortschreitender Digitalisierung kommt der technischen Vermitteltheit politischer Prozesse zwar insbesondere im Hinblick auf neue Plattformen (Lovink 2017; Gerbaudo 2019), Formen der Repräsentation (Keane 2011; Hofmann 2019a; Urbinati 2019) und Techniken der Sortierung und Kuratierung (Yeung und Lodge 2019; Amoore 2020; Rieder 2020) erneute Aufmerksamkeit zu. Die Bearbeitung dieser Fragestellungen nach den Auswirkungen digitaler Technologie bleibt jedoch trotz elaborierter technikpragmatischer Konzeptionen auf die Ebene der Performanz, also die konkreten Formen und Resultate der Wechselwirkung von Technik und Gesellschaft, beschränkt (vgl. Hofmann 2019b). Das dialektische Verhältnis zwischen technischer Ermöglichung und dem vorgestellten, inneren Raum von Machbarkeit und Verfügbarkeit bleibt so bisher nur wenig reflektiert.

Mit den Begriffen Hubigs basiert Politische Theorie jedoch, wenn sie das spezifisch Neue der digitalen Konstellation zu fokussieren versucht, in besonderem Maße auf dem Möglichkeitsraum technisch vermittelter, politischer Handlungen. Während die empirische Analyse eines einzelnen Sachverhalts mit der Performanz von Technik beschäftigt ist, zielen die normative Verortung der Analyseergebnisse, deren gesamtgesellschaftliche Einordnung oder die Problematisierung möglicher Ursachen und Folgen stets auf Verallgemeinerung und die Identifikation pfadabhängiger Entwicklungspotenziale. Zwar ist es dabei auch die Performanz, welche den Möglichkeitsraum über Einschränkungs- und Ermöglichungserfahrungen dialektisch mit formt. Die politiktheoretische Deutung von Technik ist jedoch auch die Analyse ihres Potenzialcharakters, wenn anerkannt wird, dass diese über den zweckgerichteten Mitteleinsatz hinaus selbst strukturierend wirkt (s. oben; dazu auch: Hubig 2006, S. 143). Insbesondere normativ ausgerichtete Theorien digitaler Öffentlichkeit treffen dabei teils starke Annahmen über die epistemischen Dispositionen der untersuchten Technik, indem sie die ihrer Nutzung zugrundeliegenden Zwecke aus theoretischen Prämissen ableiten. Dies betrifft nicht nur die notwendige Einschränkung ihres Untersuchungsgegenstands auf einzelne Handlungsaspekte, etwa in der Annahme, dass Akteur:innen digitale Medien zum Zweck der politischen Willensbildung nutzen. Es betrifft vor allem die Annahme, dass die (häufig aus prä-digitalen Theorien abgeleiteten) Zwecke der Techniknutzung durch neue technische Möglichkeitsräume selbst keinerlei Veränderungen unterliegen. Dies ist etwa dann der Fall, wenn davon ausgegangen wird, dass digitale Medien für dieselben Zwecke, auf dieselbe Art und mit denselben Intentionen genutzt werden wie analoge Medien der Meinungs- und politischen Willensbildung. So wird verkannt, inwieweit sich im Medium digitaler Technik neue politische Handlungsformen und Rationalitäten herausbilden, welche nach einer Neudefinition der spezifischen Merkmale politischer Öffentlichkeiten in der digitalen Konstellation verlangen. Wird, im Gegenteil, jedoch von einer einseitigen Adaption der technischen Möglichkeiten ausgegangen, 
laufen Theorien Gefahr, eine technikdeterministische Deutung anzulegen - etwa, wenn das Potenzial algorithmischer Filterung notwendig zu einer eingeschränkten Informationsdiät zu führen scheint, ohne die Möglichkeit ihrer gezielten Nutzung für spezifische Zwecke in Betracht zu ziehen. In beiden Fällen wird nicht ausreichend reflektiert, inwiefern epistemische Dispositionen und technischer Möglichkeitsraum sich dialektisch strukturieren und die korrelativen Zweck-Mittel-Relationen mit technologischem Wandel so ebenfalls wandelbar werden. So zeigt sich einerseits das epistemische Dilemma, durch welches die Analyse Gefahr läuft, ihren Gegenstand zugunsten einer theoretischen Anschlussfähigkeit zu verkennen (vgl. Alexander 1990). Andererseits zeigt sich das Problem der Singularität, bei welchem eine technikdeterministische Interpretation den digitalen Wandel als autonome, akteursunabhängige Kraft verortet (Kaufmann und Jeandesboz 2017). Wollen Theorien digitaler Öffentlichkeit jedoch der Spezifizität digitaler Technologien gerecht werden, müssen die der Technologie zugrundeliegenden epistemischen Dispositionen freigelegt und gegebenenfalls mit jenen durch die Theorie vorgegebenen in kritische Reflexion gesetzt werden. Wie sich zeigen wird, gilt dies insbesondere dann, wenn empirische Studien herangezogen werden, um das Potenzial von Technik anhand ihrer Performanz analytisch zu erschließen. Hubigs differenzierter Technikbegriff hält Ressourcen bereit, um dieses Verhältnis von Technik und Theorie in der digitalen Konstellation genauer zu fassen. So ist es gerade Technik als Weltbezug, der Mittel bereitstellt, welche die Umsetzung und Adaption von Zwecken ermöglichen, jedoch nicht bedingen. Es ist eine Fokussierung dieser Korrelativität - nicht Kausalität von Mittel und Zweck, epistemischer Disposition und technischem Möglichkeitsraum, die eine präzise Analyse der Phänomene der digitalen Konstellation ermöglicht. Im Folgenden wird dies anhand einer Kritik des Technikdeterminismus, der empirischen Untersuchung digitaler Phänomene sowie des Theorieperspektivismus veranschaulicht. Als Beispiele dienen dabei jeweils Thesen und Beiträge zur Debatte um digitale Öffentlichkeiten.

\subsection{Technikdeterminismus}

An einem Beispiel aus der Theorie digitaler Öffentlichkeiten zeigt sich besonders, wie die spezifische Wirkung technischer Erwartungen den epistemischen Möglichkeitsraum auch in der Theoriebildung mitstrukturiert. Wie Bruns (2019b) bemerkt, halten sich Theoretisierungen von Filterblasen, Echokammern und Algorithmen als Helfershelfer antidemokratischer Akteur:innen trotz fehlender empirischer Belege hartnäckig in der Diskussion um die politischen Effekte des Internets. Der technisch vermittelte (äußere) Möglichkeitsraum einer potenziellen Fragmentierung wird positivistisch interpretiert, indem er die epistemischen Dispositionen (den inneren Möglichkeitsraum) nicht nur informiert, sondern bestimmt. Das dialektische Verhältnis innerer und äußerer Medialität des Möglichkeitsraumes, welches epistemische Dispositionen und technische Machbarkeit gegeneinander abwägt, bleibt unreflektiert und „kippt“ zugunsten einer Bestimmung der Dispositionen durch die Technik. Nicht nur betrifft dies im Falle der Filterblasen und Echokammern die politische Diskussion um die Regulierung digitaler Öffentlichkeiten, es verstellt auch den Weg zu einer fundierteren Diskussion über die Ursachen der beschriebenen Phänomene: 
If there is a filter at all, then, it is not the algorithmic filter postulated by the "filter bubble“ concept, which prevents us altogether from seeing „different" content that runs counter to our own worldviews - rather, the more critical filter exists (more weakly formed perhaps in the societal mainstream, more strongly developed on the extreme fringes) in our heads, and variously leads us to adopt dominant, negotiated, and oppositional stances (cf. Hall 1980) towards the information we encounter from a multitude of sources in our daily engagement with a hybrid, multifaceted, multi-platform media environment. (Bruns 2019b, S. 10)

Wird die Kontingenz des Möglichkeitsraumes von Technik, welcher Handlungen zwar nach Maßgabe der ihm eigenen Regeln ermöglicht, jedoch nicht bedingt, vernachlässigt, läuft die Analyse so Gefahr, in Technikdeterminismus zu verfallen. Die Vorstellung technisch vermittelter Fragmentierung als notwendig eintretendes Ereignis strukturiert so die von der Analyse identifizierten epistemischen Dispositionen. Eine Gegenthese formuliert weiter Bruns, indem er die Ursache der Fragmentierung nicht in den Algorithmen, sondern bei den sie nutzenden Akteur:innen sucht. Mit Hubig formuliert: Technische Systeme ermöglichen die Umsetzung von Handlungen auf Grundlage ihnen äußerer epistemischer Dispositionen. Auf der Ebene der Performanz ermöglichen es Algorithmen so, Informationsflüsse auf die eigene Weltsicht anzupassen, insoweit es den epistemischen Dispositionen der Nutzer:innen entspricht. Das technische System, das Informationsströme so kuratiert und als ein Teil der digitalen Öffentlichkeit interpretiert werden kann, ermöglicht die kontingente Aktualisierung spezifischer Handlungen - sie bedingt sie nicht. Und es lässt sich ergänzen: Diese Handlungen werden nur in dem Maße umsetzbar, wie sie als technisch möglich erscheinen. Gerade die Vorstellung technischer Möglichkeit ist es jedoch, welche nicht nur die Techniknutzung, sondern auch eine theoretische Verortung von Technik mitstrukturiert. Es ist erst die Reflexion dieses dialektischen Verhältnisses von (inneren) epistemischen Dispositionen und technisch vermitteltem (äußeren) Möglichkeitsraum, welche es gestatten kann, die ermöglichten Handlungen zu antizipieren, ohne die epistemischen Dispositionen, die sie anleiten, aus dem Blick zu verlieren.

\subsection{Die spezifische Messbarkeit digitaler Phänomene}

Diese Überlegungen gelten insbesondere auch für den historischen Vergleich. Soll dieser der Identifikation des (wie auch immer gearteten) transformativen Charakters digitaler Technologie dienen, muss er, will er sich auf empirische Erkenntnisse stützen, deren epistemische Grundlagen sowohl im Sinne der Dispositionen (Was wird warum untersucht?) als auch im Sinne der Verfügbarkeit von Messinstrumenten (Wie kann und konnte es untersucht werden?) reflektieren. Und diese Reflexion muss sowohl für das neue, digitalisierte Phänomen als auch den War-Zustand prädigitaler Vergangenheit erfolgen, wenn eine Idealisierung dieser Vergangenheit als epistemischer blinder Fleck der Messbarkeit vermieden werden soll.

Dies betrifft, um bei dem Beispiel zu bleiben, insbesondere die Nachweisbarkeit von Echokammern auf sozialen Medien. Nicht nur differenziert sich deren Bewer- 
tung je nach demokratietheoretischem Ausgangspunkt. Auch ist ihre empirische Evidenz deutlich abhängig vom politischen, nationalen und zeitlichen Kontext - um von der Repräsentativität selbst-selektierter Nutzer:innen auf sozialen Medien ganz zu schweigen (Rau und Stier 2019). Gleichzeitig bleibt ein substanzieller Nachweis, dass analoge Öffentlichkeiten in prä-digitalen Zeiten grundlegend weniger fragmentiert waren und sind, bisher noch aus. Dies bedingt sich nicht nur durch das Problem einer retrospektiven Messung, sondern auch durch den Umstand, dass die spezifischen Beschränkungen der technischen Vermitteltheit dessen, was gemeinhin unter einer analogen „öffentlichen Meinung“ mit ihren bilateralen Gesprächen, Umfragen und Leitartikeln verstanden wird, eine Messung nicht in demselben Maße zulässt wie das Medium digitaler Technik. Der empirische Nachweis von fragmentierten digitalen Öffentlichkeiten (bzw. die Erwartbarkeit eines solchen Nachweises) baut hingegen auf den Möglichkeiten auf, diese Handlungen im digitalen Raum nachzuvollziehen und zu analysieren. So lässt sich danach fragen, welche Phänomene durch die Möglichkeit ihrer Messung überhaupt erst sichtbar werden und wie die Auswahl von Daten und Methoden die Ergebnisse mitbestimmt. ${ }^{5}$ Es ist also die (implizite) Konzeptualisierung von Technik auf Grundlage ihres (spezifisch technisch vermittelten) Möglichkeitsraums, welche eine Analyse von Technik in ihrer Performanz dialektisch mitstrukturiert. Gleichzeitig sind es gerade die in der empirischen Analyse freigelegten spezifischen Einschränkungs- und Ermöglichungserfahrungen der Performanz eines technischen Systems, welche in der Verallgemeinerung und möglichen normativen Verortung explizit die Möglichkeitsräume von Machbarkeit und Verfügbarkeit informieren. Über die korrelativen Mittel-Zweck-Relationen epistemischer Dispositionen und technischen Möglichkeitsraums lässt sich jedoch reflektieren, welche Rolle der spezifisch technischen Vermitteltheit der Analyse - nicht nur der Nutzung - technischer Systeme zukommt.

So scheint die zunehmende Polarisierung ein Phänomen zu sein, welches mit Digitalisierung zumindest einhergeht. Es fehlt jedoch der eindeutige Nachweis, dass sie durch diese bedingt ist. Stattdessen ist es möglich, dass Phänomene der Fragmentierung zwar durch digitale Technik sichtbar werden und sich in ihnen teilweise realisieren, ihre Ursache jedoch in den epistemischen Dispositionen gesucht werden muss, welche die Nutzung dieser Technik informieren - etwa langanhaltende gesellschaftliche Dekohäsionsprozesse, welche ein Bedürfnis nach Gemeinschaft wecken, das sich in virtuellen Räumen auf neue Art entfalten kann. Dass die entstehende Fragmentierung als genuin oder gar ursächlich digital eingeordnet wird, ist dabei nicht zuletzt auf die spezifische, technisch vermittelte Messbarkeit dieser Phänomene im digitalen Raum zurückzuführen. Wollen Theorien digitaler Öffentlichkeit für die Einordnung digitaler Phänomene also auf empirische Studien zurückgreifen, müssen sie nicht nur die spezifische Sichtbarmachung von Dynamiken durch digitale Technik, ihre Datengrundlage und Analysetechniken reflektieren. Sie müssen auch Konzepte entwickeln, wie mittels digitaler Analysetechniken sichtbar gemacht werden kann, welche epistemischen Positionen der Nutzer:innen sich im Zusammenspiel

\footnotetext{
5 Diese Überlegungen schließen daran an, was in der Methodendiskussion der Sozialwissenschaften als Paradigmenwechsel, respektive epistemische Herausforderung, angesichts der Verfügbarkeit umfassender digitaler Daten diskutiert wird (Kitchin 2014; Resnyansky 2019; Kinder-Kurlunda 2020).
} 
von Erwartung an Technik und Realisierung von Handlungen mittels Technik realisieren. Erst so kann auf jene zugrunde liegenden gesellschaftlichen Veränderungen rekurriert werden, welche mit der, nicht durch die, Digitalisierung eintreten.

\subsection{Theorieperspektivismus}

Die Kritik einer durch die technischen Möglichkeiten digitaler Phänomene strukturierten Epistemologie und Theorie kann jedoch nur einen ersten Schritt der Reflexion darstellen. Parallel muss danach gefragt werden, wie die epistemischen Dispositionen spezifischer Theorien und ihrer Deutungsangebote - ihr vorgestellter, ,innerer“ Raum von Machbarkeit und Verfügbarkeit (Hubig 2006, S. 121) - erst definieren, was unter den Bedingungen des technisch Möglichen als Ursache identifiziert werden kann. Innere (epistemische) und äußere (technisch vermittelte) Medialität des Möglichkeitsraumes stehen für Hubig in einem dialektischen Verhältnis, das sich in der Handlung und den dabei entstehenden Irritationen aktualisiert (s. oben). So ist es gerade die Materialität von Technik, welche als „Spur“ unerwartete positive oder negative Effekte hinterlässt und so immer wieder neu definiert, was als möglich erscheint. Nachdem oben die Gefahr einer einseitigen Überhöhung der äußeren Medialität des Möglichkeitsraumes von Technik in Form von Technikdeterminismus reflektiert wurde, muss nun die Gefahr einer Auslassung jener Materialität zugunsten einer Überhöhung seiner inneren Medialität behandelt werden. Eine solche einseitige Strukturierung des Möglichkeitsraums durch die epistemischen Dispositionen möchte ich mit dem Begriff Theorieperspektivismus benennen. Ronald Giere weist in seinem Programm des wissenschaftlichen Perspektivismus darauf hin, dass Modelle und Theorien nicht am Maßstab der Wahrheit im Sinne einer (unerreichbaren) Deckungsgleichheit von Modell und Realität, sondern lediglich anhand ihrer (zweckgebundenen) Ähnlichkeit mit einer wie auch immer gearteten Realität gemessen werden können (Giere 2006, S. 64-67). Ebenso spricht Hubig von Modellen als ,pragmatische[n] Abstraktionen“ im Sinne einer sinnvollen Vereinseitigung (Hubig 2006, S. 199). Es soll mit dem Begriff des Theorieperspektivismus also keineswegs in Abrede stehen, dass eine Fokussierung auf theoriebedingt hervorzuhebende Aspekte der Potenzialität von Technik und technischen Systemen sowohl zielführend im Sinne des Erkenntnisgewinns als auch zweckdienlich im Rahmen der Analyse sein kann. Ich möchte dennoch den Fokus auf das Verhältnis von Theorie als einem gefassten Set epistemischer Dispositionen gegenüber dem Raum technischer Möglichkeiten lenken, um die Theoretisierung von Technik als „,moving target“ in ihrer Spezifität im Sinne einer ausreichenden Realitätsähnlichkeit zu gewährleisten. Erst eine solche Reflexion des Möglichkeitsraums kann eine Einordnung der Performanz von Technik informieren, welche nicht Gefahr läuft, technische Phänomene theoretisch zu überformen und so in ihrer Materialität zu verkennen.

Bei Betrachtung der Theoretisierung digitaler Öffentlichkeit fällt auf, dass neben der oben genannten technikdeterministischen Vereinnahmung eine Tendenz vorherrscht, technische Neuerungen im Rahmen vorherrschender Paradigmen zu interpretieren und so starke Annahmen über die epistemischen Dispositionen der Techniknutzung zu treffen. Dies betraf und betrifft sowohl den Diskurs um die normative Einschätzung des Internets (Dahlberg 2011), als auch die Interpretation durch Di- 
gitalisierung im Wandel befindlicher politischer Öffentlichkeiten (Rasmussen 2014; Ritzi 2019). Je nach demokratietheoretischer Position kann eine zunehmend fragmentierte Öffentlichkeit so, aus agonaler Sicht, sowohl als Bedingung der progressiven Anfechtung hegemonialer Diskurse, als auch, aus deliberativer Perspektive, als existenzielle Bedrohung für die normative Basis der Demokratie gelten (Kaya 2020). Unterschiedliche Auslegungen derselben technischen Systeme sind nicht zwingend problematisch, weisen sie doch auf unterschiedliche theoretische Prämissen hin, welche die Grundlage für eine historische und kontextuale Einordnung bieten können. Neben der oben genannten Problematik historischer Vergleichbarkeit digitaler Technik aufgrund ihrer spezifischen Messbarkeit sei jedoch auch auf das hingewiesen, was Borucki et al. (2020) als die „Anerkennung digitaler Funktionslogik“ benennen:

[Die These], dass eine adäquate Theoretisierung und Auseinandersetzung mit der Digitaldemokratie nur funktionieren kann, wenn man ihre neuen Funktionslogiken anerkennt, statt umgekehrt, die bekannten Konzepte aus der vordigitalen Zeit lediglich als ,,verstärkt“ oder , abgeschwächt“ durch die Digitalisierung zu verstehen. (Borucki et al. 2020, S. 360)

Es sind gerade die epistemischen Dispositionen, welche, wenn sie aus theoretischen Prämissen abgeleitet werden, Gefahr laufen, die Zwecke und Rationalitäten prä-digitaler Strukturen unkritisch auf die Analyse digitaler Technologie zu übertragen, anstatt deren Veränderlichkeit anzuerkennen. Die Konzeptualisierung von Technik als strukturiertem Möglichkeitsraum, welcher sich im dialektischen Verhältnis von epistemischen Dispositionen einerseits und technischer Machbarkeit und Verfügbarkeit andererseits aktualisiert, erlaubt es hingegen, deren korrelatives Verhältnis zu fokussieren. Anstatt ein technisches System unter bereits gesetzten epistemischen Dispositionen zu lesen und ihm so eine Interpretation gewissermaßen „,̈̈berzustülpen“, kann eine Zerlegung des Verhältnisses von Theorie und Technik gewährleisten, dass die Materialität der Technik als ihre theorieunabhängige Widerständigkeit identifiziert wird, welche ihrerseits die Rationalitäten und Zwecke der Techniknutzung formt, jedoch nicht bestimmt. In einem zweiten Schritt kann dann gefragt werden, inwiefern diese Materialität nach einer Adaption der theoriebedingt unterstellten epistemischen Dispositionen verlangt, um eine gegenstandsangemessene Theoretisierung zu gewährleisten. Erst so kann die Analyse der Performanz von Technik konkrete Phänomene theoretisch präzise einordnen und letztlich auch normativ bewerten.

So erscheint es zunehmend wahrscheinlicher, dass es sich bei der Digitalisierung politischer Prozesse weniger um einen technikinduzierten „historic sea change“ (Keane 2011, S. 212) handelt, als um eine graduelle Adaption bestehender Rationalitäten, Techniken und Erwartungen an eine stets im Wandel befindliche digitale Infrastruktur (Jungherr et al. 2020). Technologischer Wandel ist somit nicht Ursache des Wandels politischer Prozesse, sondern dessen Ermöglicher unter spezifischen Bedingungen - eine Veränderung der technischen Medialität, deren spezifische Formen erst Gegenstand der Analyse sein können. Dieser Sachverhalt wurde bereits unter dem Begriff des Technikdeterminismus diskutiert. Gerade ein mangelndes Bewusstsein für die Korrelativität der Zwecke und (technischen) Mittel lässt technologischen Wandel jedoch als besonders einseitigen Treiber gesellschaftlichen Wandels 
erscheinen und unterschlägt, dass sich die Rationalität politischen Handels ebenso graduell an ihren technischen Möglichkeitsraum anpasst wie sie ihn formt. Am Beispiel der Filterblasen zeigt sich so besonders deutlich, wie das Problem mangelnder Spezifität, welches häufig mit Technikdeterminismus einhergeht, mit dem ,epistemischen Dilemma“ und dem Problem des Theorieperspektivismus gemeinsam auftreten kann: Vermeintlich notwendig eintretende Filterblasen werden anhand theoretischer Kategorien verortet, welche die Spezifität digitaler Technik nicht ausreichend berücksichtigen. Die Vor- oder Nachteile digitaler Phänomene werden so anhand ihrer Deckungsgleichheit mit (häufig idealisierten) analogen Phänomenen und Rationalitäten bewertet, ohne zu reflektieren, inwieweit digitale Technik nach einer Neuformulierung dessen verlangt, was politische Öffentlichkeit in modernen Gesellschaften konstituiert (etwa bei: Dahlberg 2011; Rasmussen 2014; Ritzi 2019; Kaya 2020). Im Gegensatz dazu verlangt ein Verständnis von technologischem Wandel als einer Erweiterung des Möglichkeitsraumes nach einer Reflexion des Verhältnisses technologischer Möglichkeiten und epistemischer Dispositionen, um analytische - und letztlich auch normative - Positionen angesichts, nicht entgegen, den Bedingungen der digitalen Konstellation zu erarbeiten. Ansonsten läuft die Analyse Gefahr, das spezifisch Neue, welches sich in der Materialität digitaler Technik niederschlägt, zugunsten eines Narrativs theoretischer Kontinuität zu verkennen.

\section{Affordanzen als Reflexionsbegriff}

Die Herstellung einer solchen Responsivität zwischen Theorie und Technik verlangt nach einem Reflexionsbegriff, welcher sich zugunsten eines praktischen Zugriffs auf den Gegenstand von der Ebene der technikphilosophischen Reflexion als ,theoretische Fokussierung auf einen Aspekt praktischer Welterschließung“ (Hubig 2006, S. 25, meine Hervorhebung) lösen muss, ohne deren begriffliches Instrumentarium aufzugeben. Reflexionsbegriff meint hier: die Herstellung von Responsivität zwischen zwei in dialektischer Beziehung stehenden Teilaspekten anhand eines die Analyse anleitenden Konzepts. Es fragt sich allerdings, auf welcher Ebene eine solche Reflexion ansetzen sollte. Technik als konkretes Phänomen kann immer nur in ihrer Form, der instrumentellen Handlung, erfahren werden. Ihre Medialität, welche in den Kategorien des inneren und äußeren Möglichkeits- und Wirklichkeitsraum analysiert wurde, muss Gegenstand einer theoretischen Reflexion bleiben. Um die Spezifität der Untersuchung zu gewährleisten, muss jedoch notwendigerweise danach gefragt werden, welche konkreten Formen ein Phänomen annimmt. Ein solcher Fokus auf die spezifische Form legt eine Verschiebung der bisherigen Diskussion um die Vermittlungsrolle von Technik im Möglichkeitsraum hin zu einer näheren Betrachtung des Wirklichkeitsraums von Technik, ihrer Performanz, nahe, da die instrumentelle Handlung stets in real existierenden technischen Systemen stattfindet. Es braucht also weiterhin einen Reflexionsbegriff, welcher die Ebene der Performanz in den Blick nimmt, ohne die Rolle von Technik in dem die Handlung informierenden Möglichkeitsraum zu leugnen.

Der Begriff der Affordanzen erscheint als geeignetes Mittel, eine solche Reflexion technischer Vermitteltheit am konkreten Gegenstand zu leisten, ohne mit den bishe- 
rigen Überlegungen zur Medialität von Technik im Widerspruch zu stehen. Davis und Chouinard (2016) weisen zu Recht auf die zahlreichen, häufig widerstreitenden Definitionen des Begriffs hin. Mit seiner dedizierten Stellungnahme zwischen deterministisch-realistischen und konstruktivistischen Positionen, in welcher er die Verflechtung sozialer Prozesse und technischer Eigenschaften betont (Hutchby 2001, S. 442), erscheint Hutchbys Definition von Affordanzen am geeignetsten, um die vorliegenden Probleme zu bearbeiten. Im Anschluss an Gibson (1979) definiert dieser Affordanzen als

functional and relational aspects which frame, while not determining, the possibilities for agentic action in relation to an object (Hutchby 2001, S. 444).

Die soziale Vermitteltheit der Affordanzen bei Hutchby, insbesondere ihre Erlernbarkeit sowie die intentionale Geschaffenheit als Design (Hutchby 2001, S. 448f.), korrespondieren mit der angeführten Konzeptualisierung der Möglichkeitsräume von Technik, welche unsere Aufmerksamkeit auf die Interpretation materieller, auf Wiederholbarkeit ausgelegter Gegebenheiten lenkt. Die Widerständigkeit jener Gegebenheiten, die Hutchby mit seinem Affordanz-Begriff hervorhebt (Hutchby 2001, S. 447), könnte ebenso als „Erfahrung der Differenz zwischen vorgestelltem und realisierten Zweck“ (Hubig 2013, S. 122) beschrieben werden. In der Handlung zeigt sich, welche realen Handlungsoptionen ein Artefakt gestattet und verweist so die vorgestellte, innere Medialität von Möglichkeits- und Wirklichkeitsraum in ihre Schranken. Der Begriff der Affordanzen steht dabei in keinem Widerspruch zum medialen Charakter von Technik: Die äußere Medialität von Möglichkeits- und Wirklichkeitsraum gibt den Raum loser Kopplungen vor, welche vorgestellt und real als die fest gekoppelten Formen instrumenteller Handlungen auftreten können. Die Analyse der Affordanzen erlaubt es, diese möglichen Formen auf Grundlage ihrer Materialität zu spezifizieren, ohne den medialen Charakter der Technik und damit die Kontingenz der Handlungen zu leugnen. Ein Hammer kann zum Einschlagen eines Nagels und zum Graben eines Loches verwendet werden, jedoch ist eine dieser beiden Verwendungsweisen - je nach Zielsetzung und Handlungsalternativen wahrscheinlicher. Und dies nicht zuletzt, da die Nutzungsweise eines Hammers sozial vermittelt und erlernt ist. Dieser Aspekt der Wahrscheinlichkeit, das „Framing“ der Handlungsmöglichkeiten (Hutchby 2001, S. 444), erlaubt es uns, zu einem gewissen Grad die Formen vorauszusagen, die das Medium einer bestimmten Technik tatsächlich annimmt. Die Analyse muss also nicht auf der Ebene aller möglichen Handlungsoptionen verbleiben, sondern kann auf jene zugespitzt werden, welche in einem spezifischen Kontext als wahrscheinlich erscheinen. Affordanzen gestatten es somit, die spezifische Medialität einer spezifischen Technologie (etwa einer digitalen Kommunikationsplattform) zu analysieren. Als Reflexionsbegriff des Wirklichkeitsraums dienen sie so dazu, die Performanz von Technik einerseits in ihrer sozialen Vermitteltheit, als Funktionsideen und Erwartungen an Technik, andererseits in ihrer Materialität, als real existierende, Handlungen ermöglichende und verunmöglichende Infrastrukturen, zu analysieren. So erlauben es Affordanzen als Bedingung intentionaler Handlungen (,,agentic action“, (Hutchby 2001, S. 444)), die Analyse auf die 
konkrete Ebene sozialer Interaktion als Gegenstand der Theorie zu fokussieren. ${ }^{6}$ Als Reflexionsbegriff des Möglichkeitsraums erlauben sie es jedoch auch, die epistemischen Dispositionen einer Theorie an ihrer äußeren Medialität - also an dem, was unter den gegebenen Bedingungen technisch möglich und wahrscheinlich erscheint zu reflektieren. Berg et al. sprechen in diesem Sinne auch von einer Generalisierbarkeit ,praktisch realisierter Affordanzen“ (Berg et al. 2020, S. 184). Der Begriff der Affordanzen verbindet somit die Ebene des Möglichkeits- und Wirklichkeitsraums und gestattet es, die epistemischen Dispositionen einer Theorie anhand der im Wirklichkeitsraum stattfindenden instrumentellen Handlungen zu reflektieren. Gleichzeitig erlauben sie es, Akteur:innen und ihre Agency innerhalb einer systemischen Darstellung von Technik zu verorten und so anschlussfähig für konkrete politikwissenschaftliche Analysen zu bleiben. ${ }^{7}$

Das Konzept der Affordanzen ermöglicht es somit, die epistemischen Dispositionen einer Theorie in der Interpretation einer konkreten Technologie zu hinterfragen und gegebenenfalls zu justieren. Darüber hinaus erlaubt die Fokussierung auf die wahrscheinlichen, sozial eingebetteten Handlungsoptionen einer Technologie es, spezifisch mögliche Formen (d. i. Handlungen) zu kontextualisieren. Die Analyse ist dem technischen Möglichkeitsraum somit nicht (epistemologisch) ,ausgeliefert“, sondern kann ihn daraufhin befragen, wie und in welchem Kontext die Formen dieser Medialität auftreten. Anstatt die epistemischen Dispositionen (den inneren Möglichkeitsraum) mit dem äußeren Möglichkeitsraum technischer Vermittlung gleichzusetzen, wie es etwa Sunstein (2017) in der Proklamation technisch möglicher, aber empirisch kaum nachweisbarer Echokammern auf digitalen Plattformen tut (Bruns 2019b), kann über die Reflexion der Affordanzen gefragt werden, welche konkreten Formen des Mediums sich wahrscheinlich manifestieren und welche epistemischen Dispositionen diese Formen der Nutzung informieren. Gleichzeitig bleibt die Theorie so anschlussfähig für empirische Analysen, welche nicht mehr nur nach den möglichen Formen, also den potenziellen Ausprägungen eines Mediums, fragen, sondern ihre tatsächlich auftretenden Formen als Manifestation konkreter (etwa kommunikativer) Handlungen im Rahmen der Möglichkeiten des Mediums untersuchen. Mittels des Konzepts der Affordanzen können tatsächlich auftretende Handlungen bereits auf Grundlage des Möglichkeitsraumes antizipiert werden, ohne in epistemologische Kurzschlüsse zu verfallen. Die Reflexion der wahrscheinlich auftretenden Handlungen in einem spezifischen Kontext erlaubt es so, die unveränderliche Materialität unseres Gegenstands zu fassen und theoretische Erwartungen entsprechend anzupassen. Erst so kann Theorie in der digitalen Konstellation eine ausreichende Spezifität in der Analyse digitaler Phänomene erreichen.

\footnotetext{
${ }^{6}$ Hutchbys Fokussierung auf menschliche Agency (vgl. Hutchby 2001, S. 444) schließt dabei an Hubigs Kritik an Luhmanns Akteursvergessenheit an: ,[...] die Autopoiesis von sozialen Systemen einschließlich ihrer technischen Optimierung, wie sie Luhmann in Teilen nachgezeichnet hat, [ist] nicht ohne diejenigen Akteure denkbar, die jeweils im Schnittpunkt zahlreicher Systeme stehen und im Zuge ihres Agierens feste Kopplungen realisieren, die das jeweilige System mit neuen Formen anreichern." (Hubig 2006, S. 183).

7 Faraj und Azad sprechen in diesem Zusammenhang auch von einer Überbrückung des ,structure-agency split“" (Faraj und Azad 2013, S. 240).
} 


\section{Skizzen einer reflexiven Epistemologie}

Um eine derart angelegte, reflexive Epistemologie für Theoriebildung in der digitalen Konstellation zu skizzieren, folgt nun eine kurze Interpretation technischer Systeme unter dem Gesichtspunkt politischer Öffentlichkeit. Als Beispiel dient die Plattform Twitter, die trotz geringer Durchdringung im deutschsprachigen Raum (Newman et al. 2019, S. 87) mit am häufigsten untersucht und insbesondere mit dem Konzept digitaler Öffentlichkeit in Verbindung gebracht wird. Dies lässt sich neben der Rezeption von Tweets durch etablierte Medien (Ross und Dumitrescu 2018) und der zunehmenden Bedeutung für Wahlkämpfe (Jungherr 2016) auch auf den vergleichsweise einfachen Zugriff auf umfassende Nutzungsdaten durch Forschende zurückführen - insbesondere, nachdem Facebook einen solchen Zugriff im Rahmen des Cambridge Analytica-Skandals quasi verunmöglichte (Bruns 2019a). Über die Affordanzen der Plattform soll ihr technischer Möglichkeitsraum analysiert und so die zugrundeliegenden epistemischen Dispositionen reflektiert werden. Diese Reflexion hilft dabei, empirische Erkenntnisse im Rahmen der Theoriebildung einzuordnen sowie theoretische Dispositionen kritisch zu hinterfragen. Erst so kann einem Mangel an Spezifizität in der Analyse vorgebeugt werden.

Bei Betrachtung der Affordanzen von Twitter wird deutlich, dass die Plattform sowohl Eigenschaften eines Informations- als auch eines sozialen Netzwerks aufweist. Die schnelle Diffusion kurzer Nachrichten (Tweets) durch Retweets wird mittels Following-Verhalten realisiert, was die Verbreitung von Informationen auf die Grundlage von Follower-Netzwerken stellt (Kwak et al. 2010). Aufgrund der Plattformarchitektur ist eine Einflussverteilung zugunsten profilierter Accounts zu beobachten (eine Power Law Verteilung), die als zentrale Weichen für die Informationsströme dienen. Gleichzeitig sind Möglichkeiten der Gemeinschaftsbildung durch eigene Follower-Netzwerke gegeben (Myers et al. 2014). Die thematische Fokussierung der Inhalte wird mittels Hashtags realisiert, welche auch netzwerkübergreifend Aufmerksamkeit generieren können (Bruns und Burgess 2015). Potenziell deliberative Prozesse werden durch öffentliche Kommentare, kommentierte Retweets und direkte Erwähnungen anderer Nutzer:innen ermöglicht. Ein Zugriff auf die Daten der Plattform durch Forschende erfolgt in der Regel durch einen von Twitter selbst bereitgestellten und damit bereits verregelten API-Zugang.

Werden diese Affordanzen unter der Perspektive einer Theorie politischer Öffentlichkeiten betrachtet, fällt auf, dass die Hybridisierung von Informations- und sozialem Netzwerk als technisches System ein Phänomen ist, welches zwar in sozialer Interaktion, analogen Räumen und Medien immer bereits realisiert wurde, jedoch mittels Digitalisierung auf neue Art und in neuem Umfang technisch realisiert werden konnte. Die persönliche Kuratierung von Informationsströmen, die durch das Folgen anderer Nutzer:innen auf Twitter realisiert wird, ist auf der technischen Seite „,analoger“ Öffentlichkeiten (im Sinne von Zeitungen, öffentlichen Debatten etc.) nur in dem Maße über räumliche und zeitliche Distanzen hinweg möglich, wie sich zwischen vorhandenen Intermediären entschieden werden kann, welche eine solche Kuratierungsleistung bereits teilweise vorwegnehmen. Mit der digitalen Öffnung der Informationskanäle als Many-to-Many-Kommunikation und dem Zugriff auf alle anderen Nutzer:innen als Informationsquellen wird die persön- 
liche Kuratierung zur zentralen Anforderung an eine heterogene Medienlandschaft (Thorson und Wells 2016). So bauen für Twitter alle weiteren Funktionen, etwa der Gemeinschaftsbildung, Deliberation und Weiterverteilung von Informationen, auf dieser Kuratierung auf. Es ist also jene Kuratierungsleistung, die durch die spezifisch digital ermöglichte Many-to-Many-Kommunikation technisch realisiert wird, welche als gegenüber analogen Öffentlichkeiten entscheidend neue Affordanz digitaler Plattformen identifiziert werden kann. Eine Affordanz ist sie in dem Sinne, dass sie sich performativ im Zwischenspiel von Nutzer:innenerwartungen und -verhalten einerseits, der Ermöglichung durch real vorhandene technische Infrastrukturen andererseits, realisiert. Auf Ebene der inneren Medialität des Möglichkeitsraums (der epistemischen Dispositionen) der Nutzenden wäre dies das Bedürfnis nach einer Kuratierung der Informationsströme; dessen äußere Medialität die Vorstellung, dass diese Leistung mittels der Plattform technisch möglich ist. Auf Ebene des Wirklichkeitsraums besteht die innere Medialität in den konkreten Kuratierungszielen (etwa spezifischen Nachrichtenkategorien), die äußere Medialität in der konkreten Infrastruktur der Plattform Twitter, welche die realen Mittel zur Umsetzung dieser Zwecke bereitstellt. In der instrumentellen Handlung der Kuratierung von Nachrichtenströmen auf der Plattform realisiert sich so der Zweck anhand konkreter Mittel. Gleichzeitig aktualisieren sich innere und äußere Medialität - etwa, indem neue Funktionen entdeckt werden, welche neue Kuratierungsziele generieren oder Erwartungen an die Funktionsweise der Plattform enttäuscht werden. So werden auch Optimierungsleistungen möglich, welche die Plattformarchitektur weiter an die Bedürfnisse und Erwartungen der Nutzer:innen anpassen.

Wird davon ausgegangen, dass die zentrale Affordanz der Selbstkuratierung notwendig in eine Fragmentierung von Öffentlichkeit mündet, wie es etwa Sunstein tut, ignoriert dies nicht nur die Performanz des Technischen in Form empirischer Evidenz, sondern auch die Agency der Nutzer:innen, welche aufgrund persönlicher Präferenzen und der (vorgestellten) Möglichkeiten ihrer Realisierung mittels des technischen Systems Handlungen realisieren. Die Übertragung der Verantwortung für die Kuratierung von Informationsflüssen auf die Nutzer:innen beinhaltet, dass lock-in Effekte abgeschlossener Informationsumgebungen möglich sind, jedoch nur in dem Maße auftreten, wie diese sich dafür (mehr oder weniger bewusst) auf Grundlage ihrer epistemischen Dispositionen entscheiden. Neue Formen der Selbstselektion von Informationsumgebungen können sich so auf digitalen Plattformen wie Twitter realisieren, bedingen sich jedoch nur in dem Maße in deren Architektur, wie diese bereits vorhandene Präferenzen ermöglicht. Die Frage ist also nicht, inwiefern digitale Plattformen neue Formen der Fragmentierung bedingt, sondern wie sich bereits vorhandene Tendenzen im Verhältnis von Nutzer:innenpräferenzen und Funktionserwartungen neu realisieren. Diese neuen Formen informationeller Selbstkuratierung müssen dabei auch im Rahmen einer multimedialen Informationsumgebung und dem sich daraus ergebenden ,multioptionale[n] Kontinuum“ (Borucki et al. 2020, S. 369) analoger und digitaler Mediennutzung zur Realisierung divergenter Zwecke eingeordnet werden. Es ist also das Konzept der Affordanzen, welches eine Rückbindung der theoretischen Konzeptualisierung von Technik an ihre spezifischen, real auftretenden Nutzungsweisen ermöglicht und so eine technikdeterministische Lesart vermeiden kann. Die Trennung in innere und äußere Medialität von Möglichkeits- 
und Wirklichkeitsraum erlaubt es dabei einerseits, auf der Ebene der Performanz aufzuschlüsseln, wie sich spezifische Affordanzen im Nutzer:innenverhalten realisieren. Andererseits ist es so möglich offenzulegen, inwieweit diese Ebene der Performanz durch die Möglichkeitsräume digitaler Technik strukturiert wird und diese wiederum dialektisch mitbestimmt. So kann reflektiert werden, inwiefern die durch eine spezifische Theorie unterstellten epistemischen Dispositionen, insbesondere die Zwecke der Techniknutzung, dem Gegenstand angemessen sind - oder eine Revision benötigen.

Bei Betrachtung digitaler Öffentlichkeiten aus einer empirischen Perspektive wird so deutlich, dass nur jene Phänomene zutage treten, die der untersuchten Plattform spezifisch zu eigen sind und im Rahmen des (im Falle von Twitter spezifisch verregelten) Zugangs zu den ihr eigenen Daten analysiert werden können. Die Kuratierungsfunktion von Twitter beruht auf der Follow-Funktion der Plattform, welche es Nutzer:innen ermöglicht, ihre spezifischen Interessen über den Konsum der Nachrichteninhalte anderer Nutzer:innen abzubilden. Diese Kernfunktion der Plattform darf jedoch nicht verkannt werden und die, netzwerktheoretisch gesprochen, $N \ddot{a}$ he der Nutzer:innen zueinander mit analogen Prozessen der Gemeinschaftsbildung gleichgesetzt werden, nur da die Plattform auch Möglichkeiten des sozialen Austauschs bietet. Die Analyse läuft sonst Gefahr, empirische Artefakte zu produzieren, welche notwendigerweise auf eine Fragmentierung nicht nur der Informationsumgebungen einer Plattform, sondern der Gesellschaft als ganzer hinweisen. Stattdessen muss danach gefragt werden, welche Dispositionen sich wie in der Plattformnutzung niederschlagen, wie diese gemessen werden können und wie sich die Phänomene einer Plattform zu einer diversen Medienlandschaft verhalten. Ohne eine solche Reflexion der Spezifität der zutage tretenden Phänomene muss jede Art des Vergleichs unzulänglich bleiben.

Das, was normativ aufgeladen als „Disruption“ oder „Turbulenz“ einer Öffentlichkeit gefasst wird (Bennett und Pfetsch 2018; Margetts 2019), welche durch den Wandel des technischen Möglichkeitsraums für ihre politische Kommunikation in abnehmenden Maß auf tradierte Intermediäre zurückgreifen muss (vgl. Eldridge II et al. 2019), lässt sich ebenso - unaufgeregter - als eine Veränderung der Medialität der technischen Systeme fassen, welche ihre eigenen Regeln der Machtverteilung produziert, ohne diese aufzulösen (vgl. Jungherr 2016; Engelmann et al. 2019). Um die tatsächlichen gesellschaftlichen Auswirkungen der untersuchten Phänomene scharf zu stellen, sollten die Spezifika des Gegenstands analysiert und dessen epistemische Dispositionen in ein kritisch-reflexives Verhältnis mit denen der angelegten Theorie gesetzt werden. Mit Blick auf Öffentlichkeitstheorien ist so nicht nur fraglich, ob eine heterogene, debattierenden Öffentlichkeit wirklich zu einer weniger polarisierten Gesellschaft beiträgt (Bail et al. 2018). Ebenso sollte gefragt werden, was die Funktionslogik von Öffentlichkeit auf digitalen Plattformen unabhängig aller Idealisierung konstituiert. Es scheint nicht das normative Ideal einer deliberierenden Gemeinschaft, sondern eher die Ratio einer Verbreitung von Informationen auf Grundlage anhaltender Interaktion zu sein, welche sich in der spezifischen Architektur der Plattform Twitter niederschlägt. Das stellt die Frage in den Raum, welcher theoretische Rahmen die für eine Einordnung digitaler Phänomene geeigneten epistemischen Dispositionen vorgibt. Das für empirische Analysen geeignetere 
theoretische Konzept scheint sich so tatsächlich weniger bei Habermas' (1990) deliberativer Öffentlichkeitskonzeption zu finden als bei Warners (2002) Konzept einer auf Zirkulation basierenden kommunikativen Öffentlichkeit, ist es doch gerade die Verbreitung von Nachrichten, nicht der Austausch von Argumenten zum Erreichen eines Konsens, welche in die Architektur der Plattform eingeschrieben ist. Eine normative Bewertung und historische Einordnung der untersuchten Phänomene soll damit keineswegs verneint werden. Theorie in der digitalen Konstellation muss, will sie eine präzise Analyse liefern, jedoch zuallererst ihren Gegenstand mit geeigneten Begriffen und Konzepten scharf stellen um dessen gesellschaftliche Auswirkungen abschätzen zu können. Dies gilt in besonderem Maße für (digitale) technische Systeme, welche als Technik ihren eigenen Möglichkeitsraum definieren und so einen spezifischen Weltbezug darstellen.

\section{Fazit}

Es wurde gezeigt, wie Technik in ihrer Medialität einen Weltbezug darstellt, welcher die Möglichkeitsräume und somit die korrelativen Mittel-Zweck-Relationen von Handlungen in der digitalen Konstellation grundlegend strukturiert. Mit dem Konzept der Affordanzen wurde ein Reflexionsbegriff eingeführt, welcher das dialektische Verhältnis zwischen epistemischen Dispositionen und technischen Möglichkeiten unter Achtung der Materialität technischer Systeme und der Agency ihrer Nutzer:innen auseinanderlegt. Anhand der digitalen Plattform Twitter wurde daraufhin demonstriert, wie eine solche Reflexion eine präzisere Benennung und Einordnung digitaler Systeme durch Theorien digitaler Öffentlichkeiten ermöglichen kann. Nicht nur sollte Politische Theorie in der digitalen Konstellation sich der herausgestellten Defizite bewusst sein, wenn diese Theorien mittlerer Reichweite als Vorüberlungen oder Argumente in größere, etwa demokratietheoretische, Arbeiten übernommen werden. Sie sollte sich ebenso der Rolle bewusst sein, die technische Möglichkeitsräume und epistemische Dispositionen im Prozess der Theoretisierung und Analyse spielen. So vertrete ich die These, dass die Reflexion von epistemischen Dispositionen und technischem Möglichkeitsraum, wenn sie mittels des Begriffs der Affordanzen an einem Gegenstand konkretisiert wird, die Theoretisierung aller technischen Systeme anleiten kann. Mögliche Themenfelder wären hier die Analyse digitaler Infrastrukturen als Systeme spezifischer Kontrolle oder ihre Auswirkungen auf den Formwandel der Demokratie im Zusammenspiel von normativer Erwartung und technischer Ermöglichung. Gleichzeitig eröffnen die dargestellten Überlegungen weitere metatheoretische und methodologische Anknüpfungspunkte, indem sie es erlauben, das Verhältnis von Theorie und Technik jenseits eines einseitigen Rationalisierungs- und Ermöglichungsprozesses zu fassen. So lässt sich nicht nur fragen, inwiefern Algorithmen der Auswertung empirische Untersuchungen digitaler Plattformen strukturieren und durch Theorieerwartungen strukturiert werden, sondern auch, inwiefern die Konzeption von Politischer Theorie in der digitalen Konstellation selbst mit diesen Prozessen in Wechselwirkung steht. In jedem Fall kann erst eine reflexive Epistemologie die Spezifität digitaler Phänomene in den Blick bekommen, indem sie die ihnen eigene, technische Strukturierung des Mög- 
lichkeitsraums anerkennt. Somit ist das hier vorgestellte Begriffsschema weniger eine ausgearbeitete Theorie von Technik denn eine Einladung, die aufgeworfenen Probleme, welche als mangelnde Spezifität und dem damit häufig einhergehenden Technikdeterminismus einerseits, als „epistemisches Dilemma“ und der Gefahr des Theorieperspektivismus andererseits identifiziert wurden, auf eine spezifische Art zu reflektieren und so präzisere Theoretisierungen der digitalen Konstellation anzuleiten. Dies gilt insbesondere dann, wenn die Ergebnisse empirischer Analysen in die Theoriebildung mit einbezogen werden sollen. Technik ist und war schon immer Weltbezug. Politische Theorie in der digitalen Konstellation darf es nicht versäumen, das spezifisch digitale dieses Weltbezugs anzuerkennen, um analytisch - und letztlich auch normativ - sprechfähig zu bleiben.

Funding Open Access funding enabled and organized by Projekt DEAL.

Open Access Dieser Artikel wird unter der Creative Commons Namensnennung 4.0 International Lizenz veröffentlicht, welche die Nutzung, Vervielfältigung, Bearbeitung, Verbreitung und Wiedergabe in jeglichem Medium und Format erlaubt, sofern Sie den/die ursprünglichen Autor(en) und die Quelle ordnungsgemäß nennen, einen Link zur Creative Commons Lizenz beifügen und angeben, ob Änderungen vorgenommen wurden.

Die in diesem Artikel enthaltenen Bilder und sonstiges Drittmaterial unterliegen ebenfalls der genannten Creative Commons Lizenz, sofern sich aus der Abbildungslegende nichts anderes ergibt. Sofern das betreffende Material nicht unter der genannten Creative Commons Lizenz steht und die betreffende Handlung nicht nach gesetzlichen Vorschriften erlaubt ist, ist für die oben aufgeführten Weiterverwendungen des Materials die Einwilligung des jeweiligen Rechteinhabers einzuholen.

Weitere Details zur Lizenz entnehmen Sie bitte der Lizenzinformation auf http://creativecommons.org/ licenses/by/4.0/deed.de.

\section{Literatur}

Alexander, Jeffrey C. 1990. Beyond the Epistemological dilemma: general theory in a postpositivist mode. Sociological Forum 5:531-544.

Amoore, Louise. 2020. Cloud ethics. Algorithms and the attributes of ourselves and others. Durham: Duke University Press. https://doi.org/10.2307/j.ctv11g97wm.

Bail, Christopher A., et al, 2018. Exposure to opposing views on social media can increase political polarization. Proceedings of the National Academy of Sciences 115:9216-9221. https://doi.org/10.1073/ pnas. 1804840115.

Bennett, W. Lance, und Barbara Pfetsch. 2018. Rethinking political communication in a time of disrupted public spheres. Journal of Communication 68:243-253. https://doi.org/10.1093/joc/jqx017.

Berg, Sebastian, Niklas Rakowski, und Thorsten Thiel. 2020. Die digitale Konstellation. Eine Positionsbestimmung. Zeitschrift für Politikwissenschaft 30:171-191. https://doi.org/10.1007/s41358-02000207-6.

Borucki, Isabelle, und Michael Oswald. 2020. Die Vision der Digitaldemokratie und die Realität - Versuch über einen Dialog. In Demokratietheorie im Zeitalter der Frühdigitalisierung, Hrsg. Michael Oswald, Isabelle Borucki, 3-15. Wiesbaden: Springer. https://doi.org/10.1007/978-3-658-30997-8_1.

Borucki, Isabelle, Dennis Michels, und Stefan Marschall. 2020. Die Zukunft digitalisierter Demokratie - Perspektiven für die Forschung. Zeitschrift für Politikwissenschaft 30:359-378. https://doi.org/10. 1007/s41358-020-00234-3.

Bruns, Axel. 2019a. After the "APIcalypse": social media platforms and their fight against critical scholarly research. Information, Communication \& Society 22:1544-1566. https://doi.org/10.1080/1369118X. 2019.1637447.

Bruns, Axel. 2019b. Filter bubble. Internet Policy Review https://doi.org/10.14763/2019.4.1426. 
Bruns, Axel, und Jean Burgess. 2015. Twitter hashtags from ad hoc to calculated publics. In Hashtag publics: The power and politics of discursive networks Digital formations, Bd. 103, Hrsg. N. Rambukkana, 13-27. Bern: Peter Lang.

Dahlberg, Lincoln. 2011. Re-constructing digital democracy: An outline of four "positions". New Media \& Society 13:855-872. https://doi.org/10.1177/1461444810389569.

Davis, Jenny L., und James B. Chouinard. 2016. Theorizing affordances: from request to refuse. Bulletin of Science, Technology \& Society 36:241-248. https://doi.org/10.1177/0270467617714944.

Eldridge, Scott A., II, Lucía García-Carretero, und Marcel Broersma. 2019. Disintermediation in social networks: conceptualizing political actors' construction of publics on twitter. Media and Communication 7:271-285. https://doi.org/10.17645/mac.v7i1.1825.

Engelmann, Ines, Andrea Kloss, Christoph Neuberger, und Tobias Brockmann. 2019. Visibility through information sharing: the role of tweet authors and communication styles in retweeting political information on twitter. International Journal of Communication 13:3569-3588.

Ess, Charles. 2018. Democracy and the Internet: a retrospective. Javnost-The Public 25:93-101. https:// doi.org/10.1080/13183222.2017.1418820.

Faraj, Samer, und Bijan Azad. 2013. The materiality of technology: an affordance perspective. In Materiality and organizing: social interaction in a technological world, Hrsg. Paul M. Leonardi, Bonnie A. Nardi, und Jannis Kallinikos, 237-258. Oxford: Oxford University Press. https://doi.org/10.1093/ acprof:oso/9780199664054.003.0012.

Gerbaudo, Paolo. 2019. The platform party: the transformation of political organisation in the era of big data. In Digital subjects: interdisciplinary perspectives on capitalism, labour and politics in the age of big data. Westminster: University of Westminster Press. https://doi.org/10.16997/book29.p.

Gibson, James J. 1979. The ecological approach to visual perception. Dallas; London: Houghton Mifflin.

Giere, Ronald N. 2006. Scientific Perspectivism. Chicago: University of Chicago Press. https://doi.org/10. 7208/chicago/9780226292144.001.0001.

Habermas, Jürgen. 1990. Strukturwandel der Öffentlichkeit: Untersuchungen zu einer Kategorie der bürgerlichen Gesellschaft. Berlin: Suhrkamp.

Hall, Stuart. 1980. Encoding/decoding. In Culture, media, language: working papers in cultural studies, 1972-79, Hrsg. Stuart Hall, Dorothy Hobson, Andrew Lowe, und Paul Willis, 128-139. London: Hutchinson.

Hofmann, Jeanette. 2019a. Mediated democracy_Linking digital technology to political agency. Internet Policy Review https://doi.org/10.14763/2019.2.1416.

Hofmann, Jeanette. 2019b. Mediatisierte Demokratie in Zeiten der Digitalisierung - Eine Forschungsperspektive. In Politik in der digitalen Gesellschaft Zentrale Problemfelder und Forschungsperspektiven, Politik in der digitalen Gesellschaft, Hrsg. Jeanette Hofmann, Norbert Kersting, Claudia Ritzi, und Wolf J. Schünemann, 27-46. Bielefeld: transcript. https://doi.org/10.14361/9783839448649-002.

Hubig, Christoph. 2006. Technikphilosophie als Reflexion der Medialität. Die Kunst des Möglichen I: Grundlinien einer dialektischen Philosophie der Technik, Bd. 1. Bielefeld: transcript.

Hubig, Christoph. 2011. Technik als Medium und „Technik“ als Reflexionsbegriff (Manuskript). https:// www.philosophie.tu-darmstadt.de/media/institut_fuer_philosophie/diesunddas/hubig/downloadshub ig/technik_als_medium_und_reflexionsbegriff.pdf. Zugegriffen: 22. Okt. 2021.

Hubig, Christoph. 2013. Technik als Medium. In Handbuch Technikethik, Hrsg. Armin Grunwald, Melanie Simonidis-Puschmann, 118-123. Stuttgart: J.B. Metzler. https://doi.org/10.1007/978-3-476-05333622.

Hutchby, Ian. 2001. Technologies, texts and affordances. Sociology 35:441-456. https://doi.org/10.1177/ S0038038501000219.

Jungherr, Andreas. 2016. Twitter use in election campaigns: a systematic literature review. Journal of Information Technology \& Politics 13:72-91. https://doi.org/10.1080/19331681.2015.1132401.

Jungherr, Andreas, Gonzalo Rivero, und Daniel Gayo-Avello. 2020. Retooling politics: how digital media are shaping democracy. Cambridge: Cambridge University Press. https://doi.org/10.1017/ 9781108297820.

Kaufmann, Mareile, und Julien Jeandesboz. 2017. Politics and "the digital": From singularity to specificity. European Journal of Social Theory 20:309-328. https://doi.org/10.1177/1368431016677976.

Kaya, Gizem. 2020. Zwischen Skylla und Charybdis? Die Zukunft der Demokratietheorie im digitalen Zeitalter. In Demokratietheorie im Zeitalter der Frühdigitalisierung, Hrsg. Michael Oswald, Isabelle Borucki, 47-81. Wiesbaden: Springer. https://doi.org/10.1007/978-3-658-30997-8_4.

Keane, John. 2011. Monitory democracy? In The future of representative democracy, Hrsg. John Keane, Wolfgang Merkel, und Sonia Alonso, 212-235. Cambridge: Cambridge University Press. https://doi. org/10.1017/CBO9780511770883.010. 
Kinder-Kurlunda, Katharina. 2020. Big Social Media Data als epistemologische Herausforderung für die Soziologie. In Soziologie des Digitalen - Digitale Soziologie?, Soziale Welt - Sonderband, Hrsg. Sabine Maasen, Jan-Hendrik Passoth, 109-133. Baden-Baden: Nomos. https://doi.org/10.5771/ 9783845295008-109.

Kitchin, Rob. 2014. Big Data, new epistemologies and paradigm shifts. Big Data \& Society https://doi.org/ $10.1177 / 2053951714528481$.

Kwak, Haewoon, Lee Changhyun, Hosung Park, und Sue Moon. 2010. What is Twitter, a social network or a news media? In Proceedings of the 19th international conference on World wide web, WWW'10, 591-600. Raleigh: Association for Computing Machinery. https://doi.org/10.1145/1772690.1772751.

Lovink, Geert. 2017. Im Bann der Plattformen: Die nächste Runde der Netzkritik, 1. Aufl., Bielefeld: transcript. https://doi.org/10.14361/9783839433683.

Luhmann, Niklas. 2015. Die Wissenschaft der Gesellschaft, 7. Aufl., Frankfurt a.M.: Suhrkamp.

Margetts, Helen. 2019. Rethinking democracy with social media. The Political Quarterly 90:107-123. https://doi.org/10.1111/1467-923X.12574.

Myers, Seth A., Aneesh Sharma, Pankaj Gupta, und Jimmy Lin. 2014. Information network or social network? the structure of the twitter follow graph. In Proceedings of the 23rd International Conference on World Wide Web, WWW'14 Companion, 493-498. Seoul: Association for Computing Machinery. https://doi.org/10.1145/2567948.2576939.

Newman, Nic, Richard Fletcher, Antonis Kalogeropoulos, und Rasmus Kleis Nielsen. 2019. Reuters Institute digital news report 2019. Oxford: Reuters Institute for the Study of Journalism.

Rammert, Werner. 2016. Technik - Handeln - Wissen: Zu einer pragmatistischen Technik- und Sozialtheorie, 2. Aufl., Wiesbaden: VS. https://doi.org/10.1007/978-3-658-11773-3.

Rasmussen, Terje. 2014. Internet and the political public sphere. Sociology Compass 8:1315-1329. https:// doi.org/10.1111/soc4.12228.

Rau, Jan Philipp, und Sebastian Stier. 2019. Die Echokammer-Hypothese: Fragmentierung der Öffentlichkeit und politische Polarisierung durch digitale Medien? Zeitschrift für Vergleichende Politikwissenschaft https://doi.org/10.1007/s12286-019-00429-1.

Resnyansky, Lucy. 2019. Conceptual frameworks for social and cultural Big Data analytics: Answering the epistemological challenge. Big Data \& Society https://doi.org/10.1177/2053951718823815.

Rieder, Bernhard. 2020. Engines of order: a mechanology of algorithmic techniques. Amsterdam: Amsterdam University Press.

Ritzi, Claudia. 2019. Politische Öffentlichkeit zwischen Vielfalt und Fragmentierung. In Politik in der digitalen Gesellschaft: zentrale Problemfelder und Forschungsperspektiven, Hrsg. Jeanette Hofmann, Norbert Kersting, Claudia Ritzi, und Wolf J. Schünemann, 61-81. Bielefeld: transcript. https://doi. org/10.14361/9783839448649-004.

Ross, Andrew R.N., und Delia Dumitrescu. 2018. "Vox Twitterati”: Investigating the effects of social media exemplars in online news articles. New Media \& Society 21:962-983. https://doi.org/10.1177/ 1461444818817313.

Seibel, Benjamin. 2016. Cybernetic Government: Informationstechnologie und Regierungsrationalität von 1943-1970. Wiesbaden: VS. https://doi.org/10.1007/978-3-658-12490-8.

Sunstein, Cass R. 2017. Republic: divided democracy in the age of social media. Princeton, Oxford: Princeton Univers. Press. https://doi.org/10.1515/9781400884711.

Thorson, Kjerstin, und Chris Wells. 2016. Curated flows: a framework for mapping media exposure in the digital age. Communication Theory 26:309-328. https://doi.org/10.1111/comt.12087.

Urbinati, Nadia. 2019. Judgment alone: cloven citizenship in the era of the Internet. In Creating political presence: the new politics of democratic representation, Hrsg. Dario Castiglione, Johannes Pollak, 61-85. Chicago, London: University of Chicago Press.

Warner, Michael. 2002. Publics and counterpublics (abbreviated version). Quarterly Journal of Speech 88:413-425. https://doi.org/10.1080/00335630209384388.

Weber, Max. 2002. Wirtschaft und Gesellschaft: Grundriss der verstehenden Soziologie, 5. Aufl., Tübingen: Mohr Siebeck.

Yeung, Karen, und Martin Lodge. 2019. Algorithmic regulation. New York: Oxford University Press. https://doi.org/10.1093/oso/9780198838494.001.0001. 